469 SURVEY ON THE USAGE OF PARP-INHIBITOR THERAPY
IN GERMANY - A NATIONAL NOGGO/JAGO-AGO
INTERGROUP STUDY

${ }^{1} \mathrm{BD}$ Nguyen-Sträuli, ${ }^{2} \mathrm{P}$ Meyer-Wilmes*, ${ }^{3} \mathrm{~J}$ Baum, ${ }^{4} \mathrm{~A}$ Kreklau, ${ }^{5} \mathrm{C}$ Buschmann, ${ }^{3} \mathrm{~S}$ Boz, ${ }^{6} \mathrm{C}$ Fotopoulou, ${ }^{7} \mathrm{M}$ Hummel, ${ }^{3} \mathrm{R}$ Chekerov, ${ }^{3} \mathrm{IE}$ Braicu, ${ }^{3} \mathrm{~K}$ Pietzner, ${ }^{3} \mathrm{~J}$ Sehouli. ${ }^{1} \mathrm{~K}$ Kinik für Gynäkologie USZ Campus, Zürich, Switzerland; ' ${ }^{2}$ Klinik für Gynäkologie und Geburtsmedizin, Uniklinik RWTH Aachen, Aachen, Germany; ${ }^{3}$ Charité - Campus Virchow Klinikum Frauenklinik, Berlin, Germany; ${ }^{4}$ Universitätsklinikum Leipzig - Klinik und Poliklinik für Frauenheilkunde, Leipzig, Germany; ${ }^{5}$ Department of Obstetrics and Gynecology, München, Germany; ${ }^{6}$ Imperial College London, UK; ${ }^{7}$ Institut Für Pathologie Charité, Berlin, Germany

\subsection{6/ijgc-2021-ESG0.408}

Introduction/Background* Poly(ADP-ribose) polymerase inhibitors (PARPi) have been approved for several tumor entities including epithelial ovarian cancer (EOC). The purpose of the study is to analyze the current implementation of BRCA testing and PARPi therapy for EOC in Germany.

Methodology The questionnaire contained 40 questions covering real life data of genetic testing and the use of PARPi. It was divided into three main parts: 1 . basic demographics of the respondents, 2. indication, counseling and selection of genetic testing, 3. approach of PARPi treatment. The questionnaire was distributed via mail from 14th August 2020 until 3rd May 2021. Statistics were descriptive. We present a subanalysis focussing on results concerning the approach of PARPi therapy.

Result(s)* 316 physicians participated in the survey. $54.9 \%$ were specialized in the field of gynecologic oncology and worked in a certified breast center $(65.7 \%)$ and/or a gynecological cancer center (68.2\%). $62.1 \%$ declared to apply PARPi on a regular basis. The majority of the respondents had practical experience with olaparib (97.3\%), followed by niraparib $(81.9 \%)$ and rucaparib $(33.0 \%)$. Criteria for selection of the appropriate PARPi were the side-effect profile $(78.7 \%)$, efficacy $(71.3 \%)$, approval status $(52.7 \%)$ and the guidelines $(51.1 \%)$. Only $11.17 \%$ considered the patients wish as relevant. Nausea/vomiting (53.2\%), thrombocytopenia (53.2\%), fatigue $(43.1 \%)$, anemia $(38.3 \%)$ and leukopenia $(30.3 \%)$ were considered the most relevant side effects in daily clinical practice. For maintenance therapy of a primary, BRCA-mutated EOC, $66.5 \%$ of the respondents would make use of a combined therapy with olaparib and bevacizumab, $23.9 \%$ would prefer olaparib only and $9.6 \%$ would choose niraparib. When deciding which PARPi to choose for maintenance therapy of a BRCA-mutated relapse of EOC, most respondents declared olaparib and bevacizumab as their first choice $(43.6 \%)$, followed by olaparib only $(33.0 \%)$ and niraparib (22.9\%).

Conclusion* PARPi have established as a standard approach for the maintenance therapy of newly diagnosed and recurrent high grade ovarian cancer. Nevertheless, education programms should be established to intensify the use of PARP-inhibition in EOC.

\section{PARP INHIBITORS MAINTENANCE THERAPY IN PLATINUM-SENSITIVE RELAPSED OVARIAN CANCER: A COMPREHENSIVE CANCER CENTRE'S EXPERIENCE}

I Moreira*, M Ferreira, AR Lopes, J Savva-Bordalo, M Abreu, S Sousa, D Pereira. Portuguese Institute of Oncology of Porto, Medical Oncology, Porto, Portugal

10.1136/ijgc-2021-ESG0.409
Introduction/Background* Olaparib and niraparib are poly ADP-ribose polymerase inhibitors (PARPis) that have shown efficacy as maintenance treatment in platinum-sensitive relapsed ovarian cancer (PSROC). The aim of this study was to assess the effectiveness and safety of maintenance PARPis in patients with PSROC, in a comprehensive cancer centre.

Methodology We retrospectively evaluated patients with PSROC treated with maintenance olaparib $(400 \mathrm{mg}$ bid, capsules or $300 \mathrm{mg}$ bid, tablets) and niraparib $(300 \mathrm{mg}$ id), who received $\geq 2$ previous lines of platinum-based chemotherapy (ChT) and had a partial or complete response to the last platinum-based regimen. Patients who received olaparib were BRCA 1/2 mutated (germline and/or somatic) and those who received niraparib were BRCA $1 / 2$ wild-type. Study endpoints were progression-free survival (PFS), overall survival (OS) and adverse events (AEs).

Result(s)* Between May 2016 and December 2020, 35 patients received maintenance PARPis (21 received olaparib and 14 received niraparib). Median age was 55 years (43-75), and all had ECOG $\leq 1$. The majority had an ovary primary tumour location (74.3\%) with serous histology (85.7\%). Most patients $(65.7 \%)$ received 2 prior platinum regimens; 22 $(62.9 \%)$ had partial response and $13(37.1 \%)$ had complete response to last platinum-based ChT. Median follow-up was 15.1 months (1.8-60.1), with 26 patients alive $(61.9 \%$ olaparib and $92.8 \%$ niraparib patients alive). Median PFS was 7.0 months (95\%CI 4.3-9.7) [median PFS for BRCA $1 / 2$ mutated and BRCA $1 / 2$ wild-type patients was $8.3(95 \% \mathrm{CI}$ 6.0-10.6) and 5.9 (95\%CI 2.3-9.4) months, respectively]. There were no differences in PFS by number of prior platinum regimens, response to last platinum-based ChT or timeto-progression after penultimate platinum-based ChT (>6-12 vs $>12$ months). Median OS was not reached. Grade $\geq 3$ AEs (anaemia, thrombocytopaenia, neutropaenia and nausea) occurred in $11(31.4 \%)$ patients $(17.1 \%$ with olaparib and $14.3 \%$ with niraparib). Treatment was suspended in 24 (68.6\%) patients: $20(57.2 \%)$ due to progression (34.3\% with olaparib and $22.9 \%$ with niraparib) and $3(8.6 \%)$ due to toxicity to niraparib (none due to olaparib). Eighteen (51.4\%) patients required dose reduction due to AEs $(28.6 \%$ with olaparib and 22.8\% with niraparib).

Conclusion* Maintenance PARPis in real-world setting is effective and has a safe toxicity profile.

\section{OVERALL SURVIVAL ASSOCIATED WITH BRCA OR ATM MUTATION STATUS IN PATIENTS WITH OVARIAN CANCER: FINDINGS FROM THE PRIOR-2 STUDY}

${ }^{1} \mathrm{G}$ Adeboyeje*, ${ }^{2} \mathrm{M}$ Sierra, ${ }^{2} \mathrm{~A}$ Bartels, ${ }^{2} \mathrm{M}$ Field, ${ }^{3} \mathrm{~S}$ Jhamb, ${ }^{3} \mathrm{~A}$ Buikema, ${ }^{4} \mathrm{~S}$ Joo. ${ }^{1}$ Merck and Co., Inc., MRL, Center for Observational and Real-World Evidence (CORE), Oncology, Kenilworth, USA; ${ }^{2}$ Optum, Commercial Analytics, Eden Prairie, USA; ${ }^{3}$ Optum, Health Economics and Outcomes Research, Eden Prairie, USA; ${ }^{4}$ Merck and Co., InC., MRL, Center for Observational and Real-World Evidence (CORE) ", North Wales, USA

\subsection{6/ijgc-2021-ESG0.410}

Introduction/Background* Many clinical guidelines recommend patients with ovarian cancer (OC) undergo mutational testing of genes involved in DNA damage repair response as a predictive marker of clinical benefit from platinum-based chemotherapy and targeted therapies. However, there is little known on the prognostic impact of BRCA or ATM mutation on the survival experience of OC patients receiving contemporary routine care in the United States. 
Methodology In this retrospective cohort study, we identified patients aged $\geq 18$ years with OC $(\geq 2$ OC diagnoses within 90 days) in Optum's de-identified electronic health record (EHR) database $(1 / 1 / 2017$ - 6/30/2020; N=16.6M total female lives). Index date was the first diagnosis of OC. Patients were stratified by BRCA/ATM status and followed for up to 24 months to assess overall survival (OS). Death was captured from the EHR and linked social security and obituary data. Two-year OS rates were evaluated using the Cox Proportional-Hazards model, adjusting for baseline demographics, comorbidities, clinical and prognostic factors.

Result(s)* Among 11,206 patients with OC, 1,901 (17.0\%) had evidence of being tested for BRCA/ATM: $616(32.4 \%)$ had BRCA/ATM mutation, 682 (35.9\%) did not BRCA/ATM mutation, and $603(31.7 \%)$ had unknown status. Patients with BRCA/ATM mutation had a mean age (SD) 59.5 (10.9) and 62.2 (12.1) years, respectively; 35.9\% of patients with BRCA/ ATM mutation had visceral metastasis at diagnosis compared with $31.8 \%$ with no mutation. Of patients with known stage at diagnosis and with BRCA/ATM mutation $(\mathrm{N}=416), 77.2 \%$ presented at stage $3 / 4$ compared with $70.6 \%$ of patients without BRCA/ATM mutation $(\mathrm{N}=503)$. Patients with BRCA/ATM mutation and no BRCA/ATM mutation were observed for a median of 705 days and 697 days, respectively. Two-year OS rates were not significantly different by BRCA/ATM mutation status (yes: $79.2 \%$ vs no: $75.4 \%, \mathrm{p}=0.13$ ); unadjusted hazard ratio (HR) 1.22 (95\% CI =0.94-1.58); adjusted HR 1.12 (95\% CI 0.85-1.47).

Conclusion* In this observational study of US patients with ovarian cancer, there was no statistically significant difference in two-year OS rates between patients with or without BRCA/ ATM mutation. Additional research is needed to evaluate the association between BRCA/ATM status and overall survival in different patient populations.

\section{COMPARISON OF PRIMARY VS. INTERVAL DEBUNKING SURGERY IN PATIENTS WITH OVARIAN CANCER MANAGED WITH ERAS PROTOCOL}

D Zouzoulas*, D Tsolakidis, C Zymperdikas, K Chatzistamatiou, C Anthoulakis, E Markopoulou, P loannidou, V Korvesi, T Mikos, T Theodoridis, E Mpili, G Pados, G Grimbizis. Aristotle University of Thessaloniki, 1st Department of Obstetrics and Gynecology, Thessaloniki, Greece

\subsection{6/ijgc-2021-ESG0.411}

Introduction/Background* Complete cytoreduction is the cornerstone of the treatment for ovarian cancer (OC). Patients are triaged either for primary debulking surgery (PDS) or neoadjuvant chemotherapy (NACT) followed by interval debulking surgery (IDS), based on the preoperative assessment. The aim of this study is to evaluate the impact of the enhanced recovery after surgery (ERAS) protocol in postoperative morbidity for both groups (PDS vs. IDS).

Methodology Retrospective analysis of women with OC from the 1st Department of Obstetrics \&Papageorgiou' Hospital (ESGO Certified Center for AOC), 2017 - 2019. Patients were triaged for PDS or IDS based on preoperative imaging and 'laparoscopic Fagotti's score'. Patient \& tumor characteristics, treatment options and follow-up information were collected. Primary outcomes were ICU admittance, post-operative complications (Clavien - Dindo classification) and duration of hospitalization.
Result(s)* 78 patients met the inclusion criteria: 40 underwent PDS and 38 IDS. The two groups had no significant difference in patients characteristics (age, Charlson comorbidity index (CCI)). Furthermore, concerning surgical outcomes PDS vs IDS group had higher surgical complexity score (SCS), blood loss and complete debulking rate, but with no statistical significance ( 5 vs. $4, p=0.1466 / 350$ vs. $300, p=0.1197 / 77.5 \%$ vs. $68.4 \%, p=0.5958$ respectively). Only the duration of the surgery was statistically significant in the PDS group (300 vs. $195 \mathrm{~min}, \mathrm{p}=0.007)$. The implementation of the ERAS protocol led to comparable results with no statistical significance for postoperative morbidity, between the two groups: The PDS group had higher ICU admittance $(17.5 \%$ vs. $2.6 \%$, $\mathrm{p}=0.9741)$, lower overall complications (15 vs. $19, \mathrm{p}=0.9741)$ and the same hospitalization $(8 \pm 3$ vs. $8 \pm 2.8$ days, $\mathrm{p}=0.3805$ ).

Conclusion* Careful preoperative selection of patients and the implementation of the ERAS program in the management of OC results in comparable postoperative morbidity between PDS and IDS, regardless of the higher SCS in the upfront surgery or the toxicity of the NACT. Further prospective studies are needed to validate these results.

\section{SURGICAL MANAGEMENT OF PREMENOPAUSAL WOMEN WITH OVARIAN CANCER}

C Martin-Gromaz, I Pelayo, V Corraliza-Galan*, E Cabezas-Lopez, C Del Valle-Rubido, MJ Pablos-Antona, D Rubio-Marin, L Abarca-Martínez, E Moratalla-Bartolome, C SanchezMartinez, J Lazaro de la Fuente. Ramon y Cajal Hospital , Obstetrics and Gynecology, Madrid, Spain

\subsection{6/ijgc-2021-ESG0.412}

Introduction/Background* Ovarian cancer (OC) is the most lethal gynaecological malignancy worldwide The standard management of ovarian cancer in premenopausal women is not clear, and much controversy remains as to whether within this group of patients some of them with advanced stages should have primary cytoreductive surgery followed by chemotherapy or neoadjuvant chemotherapy followed by interval cytoreductive surgery. There is increasing evidence that the patients gain the most benefit from surgery are those with no residual disease at the completion of surgery (R0 resection).

Methodology Retrospective analysis of women under 45 years old diagnosed of epithelial and non-epithelial ovarian cancer during the last 10 years.

Result(s)* 25 women under 45 years with OC were reviewed. Mean age at diagnosis was 36.27 years (SD 5.77; min:21, max: 43). Most of the tumors $(52 \% \mathrm{~N}: 13)$ were epithelial serous OC (Clear cells: 20.0\% N:5; Endometrioid: $12.0 \mathrm{~N}: 3$; Mucinous: $8.0 \% \mathrm{~N}: 2$; Endodermal sinus: 4.0\% N:1; Granulosa cell: $4.0 \% \mathrm{~N}: 1$ ). Most of the patients were diagnosed in advanced tumoral stages (III-IV: 68.0\%, N:17). Only 7 patients received neadyuvant quemotherapy $(28 \%)$ previous to surgery. Surgical cytoreduction was made by laparotomy in most cases $(84.0 \% \mathrm{~N}: 21)$ preferred to laparoscopy (N:4, $16.0 \%)$. Complete surgical cytoreduction was achieved in $82.0 \%$ of cases $(\mathrm{N}: 19)$. Surgical approach included: bilateral oophorectomy $(96.0 \%, \mathrm{~N}: 24)$, hysterectomy $(82.0 \% \quad \mathrm{~N}: 19)$, omentectomy $(96 \% \quad \mathrm{~N}: 24)$, appendicectomy $(60.0 \% \quad \mathrm{~N}: 15)$, peritoneal biopsy $(92.0 \% \mathrm{~N}: 23)$, peritonectomy $(60.0 \%$ $\mathrm{N}: 15)$, liver resection $(40.0 \% \mathrm{~N}: 10)$, intestinal resection (32.0\% N:8), splenectomy (N:4), colecistectomy (N:2). Pelvic and paraaortic linfadenectomy was performed in $56.0 \%$ of 